\title{
Vulnerabilidade dos profissionais da saúde no contexto da pandemia por COVID-19
}

\author{
Vulnerability of health professionals in the context of the pandemic by COVID-19 \\ Vulnerabilidad de los profesionales de la salud en el contexto de la pandemia por COVID-19
}

\author{
Maressa Ferreira de Alencar Rocha \\ ORCID: https://orcid.org/0000-0003-3014-9490 \\ Faculdade de Ciências Médicas da Paraíba, Brasil \\ E-mail: maressaalencar12@gmail.com \\ Marília Norões Viana Gadelha \\ ORCID: https://orcid.org/0000-0001-9940-3536 \\ Faculdade de Ciências Médicas da Paraíba, Brasil \\ E-mail: marilianoroes@gmail.com \\ Anna Luiza Fragoso Guimarães Costa \\ ORCID: https://orcid.org/0000-0002-4604-9964 \\ Faculdade de Ciências Médicas da Paraíba, Brasil \\ E-mail: annaaluizafgc@gmail.com \\ Bianca de Oliveira Bernardes \\ ORCID: https://orcid.org/0000-0002-0855-4409 \\ Faculdade de Ciências Médicas da Paraíba, Brasil \\ E-mail: bia.oliveira.bernardes@gmail.com \\ Lívia de Castro Mestre \\ ORCID: https://orcid.org/0000-0001-6508-3888 \\ Faculdade de Ciências Médicas da Paraíba, Brasil \\ E-mail: liviadecastromestre@gmail.com \\ Fabiana Medeiros de Brito \\ ORCID: https://orcid.org/0000-0003-3824-0168 \\ Faculdade de Ciências Médicas da Paraíba, Brasil \\ E-mail: fabianabrito_@hotmail.com
}

\begin{abstract}
Resumo
A COVID-19 é uma doença declarada pandêmica pela Organização Mundial de Saúde, responsável por causar desde manifestações gripais a síndrome respiratória aguda grave nos infectados. Tendo em vista a constante exposição e risco de contaminação dos profissionais da saúde, esse estudo teve como objetivo caracterizar a produção científica acerca da vulnerabilidade dessa população no contexto da pandemia da COVID-19. Trata-se de uma revisão integrativa da literatura cuja busca foi realizada em 25 de abril de 2021, na Biblioteca Virtual em Saúde. Para viabilizar a coleta dos dados, foram utilizados os descritores "pessoal da saúde", "infecções por coronavírus" e "risco", conectados pelo operador booleano AND, obtendo como resultado 545 artigos. Em seguida foram aplicados filtros resultando em 42 artigos. Prosseguindo com as exclusões pertinentes foi selecionada uma amostra de 22 artigos, que, após análise das publicações, foram subdivididos em duas categorias temáticas. Após a conclusão da busca e análise foi constatado que os profissionais de saúde atuando na linha de frente contra a COVID-19 estão mais suscetíveis tanto à contaminação pela doença quanto à apresentarem consequências secundárias como ansiedade, distúrbios do sono, transtornos psicológicos e depressão, como efeito principalmente da escassez de equipamentos de proteção individual adequados.
\end{abstract}

Palavras-chave: Biossegurança; COVID-19; Saúde do trabalhador; Vulnerabilidade em saúde.

\begin{abstract}
COVID-19 is a pandemic disease according to the World Health Organization, responsible for causing flu manifestations and severe acute respiratory syndrome in those infected. In view of constant exposure and infection risk of health professionals, this study aimed to describe the existent scientific publications on the vulnerability this population faces in the pandemic context. It is an integrative literature review whose search was fulfilled on the 25 th April of 2021, in the Virtual Library of Health. The designated descriptors to perform the search were "health personnel", "coronavirus infections" and "risk", associated by the boolean operator AND, leading to 545 results. Applying the filters 42 articles remained. In sequence of proper exclusions, the final sample contempled 22 articles, which were analysed and subdivided into two thematic categories. Following the analysis and search conclusion it was possible to state the major susceptibility of health professionals in the front line against COVID-19 to be infected
\end{abstract}


by the disease or develop other symptoms like anxiety, psychological or sleep disorders and depression, especially as a repercussion of personal protective equipment lack.

Keywords: Biosecurity; COVID-19; Worker's health; Health vulnerability.

\begin{abstract}
Resumen
El COVID-19 es un aliado pandémico declarado por la Organización Mundial de la Salud, responsable de causar desde manifestaciones de gripe hasta síndrome respiratoria aguda severa en los infectados. En vista de la constante exposición y riesgo de contaminación de los profesionales de la salud, este estudio tuvo como objetivo caracterizar la producción científica acerca de la vulnerabilidad de esta población en el contexto de la pandemia del COVID-19. Se trata de una revisión integradora de la literatura cuya búsqueda se realizó en 25 de abril de 2021 en la Biblioteca Virtual de Salud. Para posibilitar la recolección de datos, se utilizaron los descriptores "salud personal", "infecciones por coronavirus" y "riesgo", conectados por el operador booleano AND, resultando en 545 artículos. Entonces, se aplicaron los filtros resultando en 42 artículos. Continuando con las exclusiones relevantes, se seleccionó una muestra de 22 artículos que, a través del análisis de las publicaciones, se subdividieron en dos categorías temáticas. Tras la conclusión de la búsqueda y análisis, se pudo verificar que los profesionales de la salud que trabajan en primera línea contra el COVID-19 son más susceptibles tanto a la contaminación por la enfermedad como a presentar consecuencias secundarias como ansiedad, trastornos del sueño, trastornos psicológicos y depresión, como efecto principalmente de la escasez de equipo de protección personal adecuado.
\end{abstract}

Palabras clave: Bioseguridad; COVID-19; Salud del trabajador; Vulnerabilidad sanitaria.

\title{
1. Introdução
}

A COVID-19 é uma doença causada pelo novo coronavírus (SARS-CoV-2), sendo declarada como pandemia pela Organização Mundial da Saúde (OMS) em março de 2020. Essa infecção causa desde manifestações gripais até quadros de síndrome respiratória aguda grave (SRAG), além de apresentar-se de maneira assintomática em alguns indivíduos. Com efeito, dentre os principais sintomas apresentados estão: tosse, febre, mialgia, fadiga, dispneia e alteração do olfato e paladar. Entretanto, principalmente em portadores de comorbidades, como endócrino e cardiopatias, a evolução dessa doença tende a ser mais grave, demandando suporte ventilatório e oxigenioterapia, com desfecho menos favorável (Xavier et al., 2020).

Por causa do alto poder de infectividade e mortalidade, a COVID-19 tem configurado uma crise humanitária com impactos nos âmbitos da saúde, social, econômico e cultural. Apesar dos avanços científicos, ainda há muitas lacunas no conhecimento e na forma de lidar com essa doença, fato que traz consequências danosas em vários níveis (Santos et al., 2020; Silva et al., 2020).

Uma das populações mais afetadas por essa pandemia foi a dos profissionais de saúde, os quais apresentam várias vulnerabilidades frente a esse contexto visto que eles estão na linha de frente de combate a COVID-19. Dentre as referidas vulnerabilidades, pode-se citar: risco de adoecimento e morte pela infecção, transmissão da doença para os familiares, falta de equipamentos individuais de proteção (EPI's) adequados, precariedade estrutural no local de trabalho, jornadas de trabalho exaustivas e esgotamento psicológico desencadeando doenças como ansiedade, depressão e síndrome de Burnout (Silva et al., 2020; Santana et al., 2020).

No que diz respeito à exposição ao vírus, até setembro de 2020 foram diagnosticados 181.886 casos de COVID-19 entre os profissionais de saúde, o que representa cerca de $4 \%$ do número total de infectado no referido período. Esse dado enfatiza o alto risco de contaminação dos profissionais de saúde, sendo os técnicos de enfermagem e enfermeiros os principais atingidos pela doença. Desse modo, salienta-se que os trabalhadores da saúde estão expostos a um risco de contaminação cerca de três vezes maior do que na população geral (Santos et al., 2020).

Não obstante, as vulnerabilidades têm emergido em sua maioria em decorrência de interrupções abruptas de planos e projetos individuais, cisão das relações cotidianas, interação social, redução dos fluxos entre comunidades e com as alterações da própria rede de vínculo socioafetivo (Sousa, Ribeiro, Oliveira \& Silva, 2020). Além disso, o sentimento de insegurança relacionado à contaminação é intensificado pela necessidade de manter-se assíduos e em constante contato com outras pessoas 
nos ambientes de trabalho. Diante dessas fragilidades, a proteção da saúde mental e física dos trabalhadores da saúde reforça o valor para garantir a continuidade dos processos de trabalho nos diversos níveis de atenção à saúde (Santana et al., 2020).

Frente ao exposto, surge a necessidade de aprofundamento desse tema para identificar problemas e subsidiar intervenções, visto que a pandemia da COVID-19 é algo atual e carente de produção científica e, especialmente em relação aos profissionais de saúde, é válido focar a atenção, pois estão entre os mais expostos às consequências diretas e indiretas dessa pandemia. Nesse contexto, o estudo tem por objetivo caracterizar a produção científica acerca da vulnerabilidade dos profissionais da saúde no contexto da pandemia por COVID-19.

\section{Metodologia}

Trata-se de uma revisão integrativa da literatura de natureza qualitativa e descritiva que teve a finalidade de identificar, analisar e sintetizar pesquisas em bases de dados eletrônicas sobre a vulnerabilidade dos profissionais de saúde diante da pandemia causada pelo novo coronavírus. Tal estudo tem o intuito de proporcionar uma compreensão integral do fenômeno analisado e contribuir para a tomada de decisões, visando melhor qualidade de vida do usuário, além de instigar o pensamento crítico necessário na prática diária (Souza, Silva \& Carvalho, 2021).

Diante do exposto, percebe-se que a revisão integrativa de literatura se constitui como um instrumento da Prática Baseada em Evidências (PBE). Nesse contexto, as seguintes etapas foram utilizadas para operacionalização dessa revisão: elaboração da pergunta norteadora; busca na literatura; coleta de dados utilizando critérios de inclusão e exclusão; análise crítica dos estudos selecionados; discussão dos resultados e apresentação da revisão integrativa. Respeitando os referidos critérios, foi elaborada a seguinte pergunta norteadora da pesquisa: Mediante as evidências científicas, quais as vulnerabilidades dos profissionais da saúde diante da pandemia da COVID-19?

A busca dos dados foi realizada em 25 de abril de 2021, através da Biblioteca Virtual em Saúde (BVS), nas seguintes bases de dados: Medical Literature Analysis and Retrieval System Online (MEDLINE), Literatura Latino-americana e do Caribe em Ciências da Saúde (LILACS), WHO IRIS e Base de Dados de Enfermagem (BDENF). Os Descritores em Ciências da Saúde (Decs) escolhidos para responder à questão foram: pessoal da saúde, infecções por coronavírus e risco. A seguir, procedeu-se à busca dos artigos, utilizando-se os três descritores ligados pelo conectivo AND. Foram adotados os seguintes critérios de inclusão para a seleção dos artigos: disponibilidade do texto completo; estar escrito em inglês, português e espanhol; ter sido publicado nos últimos 5 anos (2016-2021) e tratar como assunto principal a saúde do trabalhador e a saúde mental. Foram excluídos estudos que não atenderam à questão norteadora e aos critérios de inclusão mencionados, além de artigos que não demonstram adequadamente o referencial teórico e metodológico e/ou rigor científico e ético.

A partir da pesquisa nas bases de dados utilizando os descritores, foram obtidos inicialmente um total de 545 artigos. Após a aplicação dos filtros, restaram 42 estudos. Ocorrendo em seguida a leitura minuciosa de cada título, resumo e artigo na íntegra, foram selecionados os artigos que se relacionavam com objetivo do estudo. Após essa leitura, 22 artigos foram escolhidos e suas informações organizadas em um instrumento de coleta de dados contendo: título do artigo, autores e ano de publicação, base de dados, objetivo do trabalho, idioma e periódico.

Posteriormente, com o instrumento pronto, foram extraídos os conteúdos abordados nos artigos de interesse dos pesquisadores. Os trabalhos foram comparados e agrupados por similaridade de conteúdo, sendo construídas duas categorias para análise.

\section{Resultados e Discussão}

Para facilitar a identificação dos estudos incluídos na revisão integrativa, a Tabela 1 apresenta um quadro-síntese. 
Tabela 1 - Quadro-síntese com as identificações dos estudos:

\begin{tabular}{|c|c|c|c|c|}
\hline Título do estudo & Autor(es), Ano & Base de dados & Idioma & Periódico \\
\hline $\begin{array}{l}\text { Prevention related to the occupational } \\
\text { exposure of health professionals } \\
\text { workers in the COVID-19 scenario }\end{array}$ & $\begin{array}{c}\text { Gallasch CH, Cunha ML, } \\
\text { Pereira LAS, Silva-Junior JS. } \\
2020\end{array}$ & LILACS & Inglês & Rev. enferm. UERJ \\
\hline $\begin{array}{c}\text { The impact of SARS-CoV-2 on the } \\
\text { mental health of healthcare workers } \\
\text { in a hospital setting-A Systematic } \\
\text { Review. }\end{array}$ & $\begin{array}{c}\text { Sanghera J, Pattani N, Hashmi } \\
\text { Y, Varley KF, Cheruvu MS, } \\
\text { Bradley A, Burke JR. } \\
2020\end{array}$ & MEDLINE & Inglês & J Occup Health \\
\hline $\begin{array}{l}\text { Mental health of healthcare workers } \\
\text { during the COVID-19 outbreak: A } \\
\text { rapid scoping review to inform } \\
\text { provincial guidelines in South Africa. }\end{array}$ & $\begin{array}{l}\text { Robertson, L J; Maposa, I; } \\
\text { Somaroo, H; Johnson, O. } \\
2020\end{array}$ & MEDLINE & Inglês & S Afr Med J \\
\hline $\begin{array}{l}\text { Construção e validação do checklist } \\
\text { para paramentação e } \\
\text { desparamentação dos equipamentos } \\
\text { de proteção individual }\end{array}$ & $\begin{array}{c}\text { Braga LM, Siman AG, Souza } \\
\text { CC, Dutra HS, Gomes AP, } \\
\text { Siqueira-Batista R. } \\
2020\end{array}$ & LILACS & Português & $\begin{array}{l}\text { Rev. enferm. Cent.- } \\
\text { Oeste Min }\end{array}$ \\
\hline $\begin{array}{l}\text { The mental health impact of the } \\
\text { covid-19 pandemic on healthcare } \\
\text { workers, and interventions to help } \\
\text { them: A rapid systematic review }\end{array}$ & $\begin{array}{l}\text { Muller AE, Hafstad EV, } \\
\text { Himmels JPW, Smedslund G, } \\
\text { Flottorp S, Stensland SO, } \\
\text { Stroobants S, Van de Velde S, } \\
\text { Vist G. } \\
2020\end{array}$ & MEDLINE & Inglês & Psychiatry Res \\
\hline $\begin{array}{l}\text { Assessment of Mental Health Factors } \\
\text { among Health Professionals } \\
\text { Depending on Their Contact with } \\
\text { COVID-19 Patients. }\end{array}$ & $\begin{array}{l}\text { Wańkowicz P, Szylińska A, } \\
\text { Rotter I. } \\
2020\end{array}$ & MEDLINE & Inglês & $\begin{array}{c}\text { Int J Environ Res Public } \\
\text { Health }\end{array}$ \\
\hline $\begin{array}{l}\text { Work-Related and Personal Factors } \\
\text { Associated With Mental Well-Being } \\
\text { During the COVID-19 Response: } \\
\text { Survey of Health Care and Other } \\
\text { Workers. }\end{array}$ & $\begin{array}{l}\text { Evanoff B, Strickland J, Dale A, } \\
\text { Hayibor L, Page E, Duncan J, } \\
\text { Kannampallil T, Gray DL. } \\
2020\end{array}$ & MEDLINE & Inglês & J Med Internet Res \\
\hline $\begin{array}{l}\text { COVID-19 pandemic from an } \\
\text { ophthalmology point of view. }\end{array}$ & $\begin{array}{l}\text { Gupta, Parul Chawla; Kumar, } \\
\text { M Praveen; Ram, Jagat. } \\
2020\end{array}$ & MEDLINE & Inglês & Indian J Med Res \\
\hline $\begin{array}{c}\text { Anti-SARS-CoV-2 antibody testing } \\
\text { in IBD helthcare professionals: are } \\
\text { we currently able to provide COVID- } \\
\text { free IBD clinics? }\end{array}$ & $\begin{array}{l}\text { Malickova K, Kratka Z, Luxova } \\
\text { S, Bortlik M, Lukas M. } \\
2020\end{array}$ & MEDLINE & Inglês & $\begin{array}{c}\text { Scandinavian Journal of } \\
\text { Gastroenterology }\end{array}$ \\
\hline $\begin{array}{l}\text { Prevenção de contágio por covid-19 } \\
\text { na exposição ocupacional em saúde: } \\
\text { scoping review }\end{array}$ & $\begin{array}{c}\text { Conegliani TV, Uehara SC da } \\
\text { SA, Magri MA. } \\
2020\end{array}$ & $\begin{array}{l}\text { BDENF - } \\
\text { Enfermagem }\end{array}$ & Português & CuidArte, Enferm \\
\hline $\begin{array}{l}\text { COVID-19 and Keeping Clean: A } \\
\text { Narrative Review To Ascertain the } \\
\text { Efficacy of Personal Protective } \\
\text { Equipment To Safeguard Health Care } \\
\text { Workers Against SARS-CoV-2. }\end{array}$ & $\begin{array}{l}\text { Sud SR, MD. } \\
\qquad 2020\end{array}$ & MEDLINE & Inglês & Hosp Pediatr \\
\hline
\end{tabular}




\begin{tabular}{|c|c|c|c|c|}
\hline $\begin{array}{l}\text { The impact of having inadequate } \\
\text { safety equipment on mental health }\end{array}$ & $\begin{array}{l}\text { Simms, A; Fear, N T; } \\
\text { Greenberg, N. } \\
2020\end{array}$ & MEDLINE & Inglês & Occup Med (Lond) \\
\hline $\begin{array}{l}\text { Brief guideline for the prevention of } \\
\text { COVID-19 infection in head and } \\
\text { neck and otolaryngology surgeons }\end{array}$ & $\begin{array}{c}\text { Boccalatte LA, Larrañaga JJ, } \\
\text { Perez Raffo GM, Teijido CA, } \\
\text { García Fornari G, Staneloni MI, } \\
\text { Figari MF. } \\
2020\end{array}$ & MEDLINE & Inglês & Am J Otolaryngol \\
\hline $\begin{array}{l}\text { COVID-19: minimising risk to } \\
\text { healthcare workers during aerosol- } \\
\text { producing respiratory therapy using } \\
\text { an innovative constant flow canopy }\end{array}$ & $\begin{array}{c}\text { Adir Y, Segol O, Kompaniets } \\
\text { D, Ziso H, Yaffe Y, Bergman I, } \\
\text { Hassidov E, Eden A. } \\
2020\end{array}$ & MEDLINE & Inglês & Eur Respir J \\
\hline $\begin{array}{l}\text { COVID-19 in cardiac arrest and } \\
\text { infection risk to rescuers: A } \\
\text { systematic review }\end{array}$ & $\begin{array}{c}\text { Couper K, Taylor-Phillips S, } \\
\text { Grove A, Freeman K, Osokogu } \\
\text { O, Court R, Mehrabian A, } \\
\text { Morley PT, Nolan JP, Soar J, } \\
\text { Perkins GD. } \\
2020\end{array}$ & MEDLINE & Inglês & Resuscitation \\
\hline $\begin{array}{l}\text { Equipamentos de proteção individual } \\
\text { para atendimento de casos suspeitos } \\
\text { ou confirmados do novo Coronavírus }\end{array}$ & $\begin{array}{c}\text { Tristão FS, Tavares DH. } \\
2020\end{array}$ & LILACS & Português & $\begin{array}{l}\text { Journal of Nursing and } \\
\text { Health }\end{array}$ \\
\hline $\begin{array}{c}\text { The Impact of COVID-19 Epidemic } \\
\text { Declaration on Psychological } \\
\text { Consequences: A Study on Active } \\
\text { Weibo Users. }\end{array}$ & $\begin{array}{l}\text { Li S, Wang Y, Xue J, Zhao N, } \\
\text { Zhu T. } \\
2020\end{array}$ & Medline & Inglês & Plubic Heatlh \\
\hline $\begin{array}{l}\text { De cuidador a paciente: na pandemia } \\
\text { da Covid- } 19, \text { quem defende e cuida } \\
\text { da enfermagem brasileira? }\end{array}$ & $\begin{array}{c}\text { Soares SSS, Souza NVDO, } \\
\text { Carvalho EC, Varella TCMML, } \\
\text { Andrade KBS, Pereira } \\
\text { SRM,Costa C. } \\
2020\end{array}$ & LILACS & Inglês & Escola Anna Nery \\
\hline $\begin{array}{l}\text { Health workers exposure risk } \\
\text { assessment and management in the } \\
\text { context of COVID-19 virus: interim } \\
\text { guidance, } 4 \text { March } 2020\end{array}$ & $\begin{array}{l}\text { World Health Organization. } \\
\qquad 2020\end{array}$ & WHO IRIS & Inglês & $\begin{array}{ll}\text { World } & \text { Health } \\
\text { Organization } & \end{array}$ \\
\hline $\begin{array}{c}\text { Covid-19: Por que a proteção de } \\
\text { trabalhadores e trabalhadoras da } \\
\text { saúde é prioritária no combate à } \\
\text { pandemia? }\end{array}$ & $\begin{array}{l}\text { Helioterio MC, Lopes FQRS, } \\
\text { Sousa CC, Souza FO, Pinho PS, } \\
\text { Sousa FNF, Araújo, TM. } \\
2020\end{array}$ & LILACS & Português & $\begin{array}{l}\text { Trabalho, Educação e } \\
\text { Saúde }\end{array}$ \\
\hline $\begin{array}{l}\text { Condições de trabalho e o impacto na } \\
\text { saúde dos profissionais de } \\
\text { enfermagem frente a Covid-19 }\end{array}$ & $\begin{array}{l}\text { Miranda FMD, Santana LDL, } \\
\text { Pizzolato AC, Sarquis LMM. } \\
2020\end{array}$ & LILACS & Português & Cogitare enfermagem \\
\hline $\begin{array}{l}\text { Time de Resposta Rápida em Saúde } \\
\text { Mental (TRRSM): protocolo de } \\
\text { atendimento psicossocial para } \\
\text { trabalhadores da saúde no contexto } \\
\text { de pandemia }\end{array}$ & $\begin{array}{l}\text { Poersch AL, Cardoso DL, } \\
\text { Ramos MZ, Lima FM, Carvalho } \\
\text { FG. } \\
2020\end{array}$ & LILACS & Português & $\begin{array}{l}\text { Clinical \& Biomedical } \\
\text { Research, }\end{array}$ \\
\hline
\end{tabular}


De acordo com os resultados encontrados foi possível constatar que a maioria dos artigos foram publicados na língua inglesa, com $16(72,72 \%)$ estudos, seguidos pela língua portuguesa com 06 (27,27\%) publicações. Apesar da inclusão através do filtro de língua espanhola, nenhum artigo publicado nesta língua foi selecionado na amostra final. Em relação ao ano de publicação, ressalta-se que todos os artigos foram publicados em 2020.

No tocante aos países das pesquisas, 08 artigos são do Brasil (36,36\% do total), caracterizando-se como o país de maior número de publicações. O Reino Unido ficou em segundo lugar com 04 artigos (13,63\%) e os Estados Unidos em terceiro, com 02 publicações, representando 9,09\% do total. O restante dos países foi responsável por 01 artigo cada (4,54\%), sendo eles: África do Sul, Noruega, Polônia, Índia, República Tcheca, Argentina, China e Suíça. Dessa forma, quanto à distribuição por continente, $11(50 \%)$ estudos foram realizados no continente americano, 08 (36,36\%) no continente europeu, $02(9,09 \%)$ no continente asiático e apenas 01 (4,54\%) no continente africano.

Foi possível observar nessa amostra uma prevalência de artigos da base Medical Literature Analysis and Retrieval System Online (MEDLINE) (59,09\%), seguida pela Literatura Latino-americana e do Caribe em Ciências da Saúde (LILACS) $(31,81 \%)$, que concentraram a maioria dos artigos. Nas bases WHO IRIS e Base de Dados de Enfermagem (BDENF) foram encontrados 02 artigos, 01 em cada, representando 4,54\% da amostra final, respectivamente.

Assim, o conhecimento produzido pela literatura investigada foi sintetizado em duas categorias temáticas: I) “Consequências da COVID-19 na saúde mental dos profissionais”; e II) “Contaminação dos profissionais da saúde: medidas de prevenção frente a COVID-19”.

\section{Categoria I - Consequências da COVID-19 na saúde mental dos profissionais}

O cenário da pandemia trouxe consigo diversas dificuldades dentre elas pode-se citar as incertezas sobre a doença e o isolamento social imposto à população, que além de precisarem enfrentar o medo da doença, precisam se manter afastadas das atividades rotineiras mesmo diante de situações financeiras, sociais e familiares desfavoráveis a isso. Tais dificuldades ocasionam consequências diretas na vida dos indivíduos como sentimento de impotência, raiva e preocupação (Miranda, Santana, Pizzolato \& Sarquis, 2020).

A maioria dos grupos populacionais vem apresentando mudanças nos estados emocionais, como pode ser observado em um estudo realizado por meio de uma plataforma digital chinesa, em que foram analisados os sentimentos dos usuários a partir de suas postagens durante a pandemia. Foi verificado que, com o passar do tempo, os internautas começaram a se expressar com mais palavras de denotação preocupante e de emoção negativa, como ansiedade, depressão e indignação, enquanto que publicações com palavras de cunho positivo como amigos e lazer diminuíram, evidenciando, portanto, piora das condições psicológicas quando associadas à pandemia (Li, Wang, Xue, Zhao \& Zhu, 2020).

Esse quadro é ainda mais notório nos profissionais de saúde, os quais, quando comparado a população geral apresentaram maiores níveis de ansiedade, depressão e insônia em vários estudos e com significância estatística, com p < 0,05 em todos (Sanghera et al., 2020). Quanto aos tipos de acometimentos psicológicos mais encontrados nos profissionais de saúde, uma revisão sistemática da literatura com 44 artigos que utilizaram instrumentos de avaliação psiquiátrica para identificar transtornos, destacando-se as seguintes alterações: depressão, ansiedade, reação de estresse agudo, transtorno de estresse pós-traumático, insônia e burnout ocupacional (Sanghera et al., 2020).

As alterações supracitadas são evidenciadas principalmente em profissionais da linha de frente, em consonância com um estudo transversal realizado na Polônia, o qual evidenciou que os profissionais de saúde que trabalhavam na linha de frente contra o COVID-19 apresentaram com maior frequência sintomas de depressão, ansiedade e distúrbios do sono (todos com p < 0,001) quando comparados aos profissionais de segunda linha, isto é, trabalhadores que não atuavam em unidades de terapia 
intensiva (UTI), unidades de doenças infecciosas ou em departamentos de emergência. Além disso, os profissionais de primeira linha eram mais prováveis de fumar e de sofrer de dislipidemia ( $\mathrm{p}<0,025$ e $\mathrm{p}<0,01$, respectivamente) que o outro grupo (Wańkowicz, Szylińska \& Rotter, 2020).

Achados semelhantes foram observados em estudo realizado em Washington, no qual foi identificado que os profissionais que trabalhavam em situações de alto risco de contaminação pelo coronavírus (UTIs, salas de emergência ou em procedimentos geradores de aerossol) eram mais propensos a sofrer de altos níveis de estresse, exaustão, burnout, depressão e ansiedade (Evanoff et al., 2020).

Quanto à análise dos subgrupos de profissionais de saúde, a maioria dos estudos mostrou que os profissionais de enfermagem possuíam maior prevalência de todos os distúrbios psicológicos quando comparados aos médicos, porém alguns estudos destacaram maior prevalência de alguns distúrbios como insônia em médicos, já em relação ao esgotamento, médicos residentes possuem maior risco que enfermeiros (Sanghera et al., 2020). Além disso, dentre os médicos, estudos mostram que algumas especialidades têm maiores riscos de exposição ao coronavírus que outras, a exemplo dos oftalmologistas, em razão da proximidade entre esses profissionais e os pacientes durante o exame clínico e da presença do vírus no fluido lacrimal (Gupta, Kumar \& Ram, 2020).

Outrossim, sabe-se que tais consequências para a saúde mental estão diretamente relacionadas a diversos fatores de risco vivenciados na pandemia, dentre eles, a exposição direta ao paciente com COVID-19 foi o mais demonstrado para todos os tipos de transtornos psicológicos, pois todos tiveram $\mathrm{P}<0,05$, exceto burnout. Outros fatores de risco particulares de cada transtorno foram: proteção pessoal insuficiente, menor experiência, falta de treinamento, falta de apoio social, menor acesso ao apoio psicológico, medo de autoinfecção, cargas horárias maiores de trabalho, falta de oportunidades para descansar, sono irregular e recursos inadequados para o quantitativo de pacientes (Sanghera et al., 2020; Robertson, Maposa, Somaroo \& Johnson, 2020; Muller et al., 2020).

Nesse contexto, enfatiza-se o impacto da falta da disponibilidade de EPI's adequados no risco dos profissionais de saúde apresentarem sintomas de transtornos mentais comuns, transtorno de estresse pós-traumático, piora da saúde geral e problemas emocionais, frente à constante pressão do trabalho, ameaça de infecção e condições de trabalho desfavoráveis (Simms, Fear \& Greenberg, 2020). Em contrapartida, destacam-se os fatores de proteção para evitar o desenvolvimento de transtornos psicológicos, em que os mais citados nos estudos foram: o suporte social, pelos familiares, amigos e conhecidos e o apoio dos serviços e supervisores onde os profissionais atuam (Sanghera et al., 2020; Robertson, Maposa, Somaroo \& Johnson, 2020).

Assim, vale ressaltar a importância de atentar para a estratificação de risco em relação ao desenvolvimento de sintomas psiquiátricos dentre os profissionais de saúde, levando em consideração as diferentes características relacionadas ao perfil dos trabalhadores, os achados clínicos e os fatores de risco apresentados por eles, no intuito de estabelecer intervenções que previnam ou auxiliem na conduta (Poersch, Cardoso, Ramos, Lima \& Carvalho, 2020).

Tais intervenções precisam partir tanto da instituição, com medidas de apoio e suporte, treinamento adequado, comunicação clara, reconhecimento de distúrbios psicológicos e encaminhamento adequado, quanto do apoio psicológico, com psicoterapia online, alerta sobre sinais de transtornos psicológicos e uso de psicotrópicos se necessário (Robertson, Maposa, Somaroo \& Johnson, 2020). Outro estudo concorda com essa linha de pensamento pois institui como outras intervenções possíveis: instituição de turnos menores e menos cansativos, linha direta de comunicação com a instituição e com psicólogos, fornecimento suficiente de equipamentos de proteção individual e instituição de horários de descanso (Muller et al., 2020). 


\section{Categoria II - Contaminação dos profissionais da saúde: medidas de prevenção frente à COVID-19}

A infecção pelo coronavírus pode se dar de forma direta ou indireta, sendo no primeiro caso através de micro gotículas geradas por espirros ou tosse de pacientes contaminados que se propagam pelo ar podendo ser inspiradas por quem esteja próximo e sem proteção adequada. Já no segundo caso essas micro gotículas permanecem nas superfícies de objetos ou locais nos quais através do contato serão levadas até o novo indivíduo (Boccalatte et al., 2020).

Os profissionais de saúde são o grupo mais exposto a COVID-19 por conta do contato prolongado com pacientes e seus líquidos corporais, principalmente gotículas de saliva e, apesar disso, existem falhas na proteção desses profissionais, os quais não podem seguir o distanciamento social como a maioria da população, pois precisam garantir os cuidados essenciais relacionados aos agravos da COVID-19. Isso leva a insalubridade no local de trabalho e maior risco de contaminação, principalmente pela falta de EPI's, pela falta de capacitação quanto à paramentação e desparamentação desses equipamentos de proteção e pela jornada de trabalho extensa. Assim, é de extrema importância prezar pelo cuidado daqueles que cuidam (Gallasch, Cunha, Pereira \& Silva-Junior, 2020; Braga et al., 2020).

Nesse viés, destaca-se especialmente a enfermagem por se tratar de profissionais que estão mais próximos do paciente e por tempo prolongado e, com isso, estão tendo repercussões diretas nessa pandemia em virtude dos riscos laborais e das condições inadequadas de trabalho. Dentre os riscos, enfatiza-se a precariedade dos serviços em que faltam recursos essenciais, como os EPIs, tornando os trabalhadores ainda mais vulneráveis ao adoecimento (Soares et al., 2020).

Os profissionais de saúde podem estar expostos a geração de aerossóis também em procedimentos como a Reanimação Cardiopulmonar (RCP), composta pelas compressões torácicas, desfibrilação e respiração e apesar de não ser bem estabelecida em consenso a geração de aerossóis durante a RCP, é preconizada a saúde do socorrista nesse momento, devendo sempre utilizar os EPIs adequados (Couper et al., 2020).

Em relação aos sintomas apresentados, um estudo relatou que de 92 profissionais de saúde examinados, 9 relataram sinais clínicos de infecção do trato respiratório superior e/ou inferior com febre e tosse nas últimas 8 semanas, 5 apresentaram falta de ar e 3 tiveram diarréia (Malickova, Kratka, Luxova, Bortlik \& Lukas, 2020).

Assim, frente ao risco aumentado que esses profissionais possuem e visando a proteção deles até mesmo para evitar que sejam vetores da doença, é preciso instituir medidas mais eficazes de proteção, como: barreira de contato para exposição prolongada a pacientes infectados através do uso de EPIs adequados e treinamento para uso correto, triagem correta dos pacientes com COVID-19 para garantir que os profissionais tomem todas as medidas de precaução recomendadas, utilização de medidas de precaução de contato adequadas para cada procedimento e afastamento de profissionais com risco para complicação (Gallasch, Cunha, Pereira \& Silva-Junior, 2020).

Outras medidas para mitigar a contaminação de profissionais de saúde pelo novo coronavírus citadas por um estudo de revisão de escopo foram: prática da higienização das mãos com água e sabão ou álcool a 70\%; controles ambientais (por exemplo, tratamento de ar especializado), realização da técnica de paramentação e desparamentação com posterior banho; utilização da telemedicina; implementação de barreiras físicas e uso de máscara pelo paciente (Conegliani, Uehara \& Magri, 2020; Sud, 2020).

Atentar para os tipos de procedimentos e o risco de contaminação a eles relacionados também é bastante importante para orientar quanto a prevenção dos profissionais, através de recomendações relacionadas à assistência ao paciente. Assim, destaca-se os procedimentos de maior geração de aerossóis, como cânula nasal de alto fluxo (HFNO), pressão positiva contínua em vias aéreas (CPAP) e ventilação não invasiva (VNI) e, nesses casos, dispositivos ou técnicas que reduzam a dispersão dessas partículas devem ser adotados (Conegliani, Uehara \& Magri, 2020). Dentre os citados, a cânula nasal de alto fluxo é a melhor opção para evitar os procedimentos mais invasivos, pois tem sido comprovadamente eficaz em reduzir a 
necessidade de intubação nos pacientes em comparação com a oxigenação tradicional, sem alteração no risco de morte ou tempo de internação, enquanto a VNI ainda possui altas taxas de falha terapêutica (Adir et al., 2020).

Não obstante, a melhoria das condições de trabalho, redefinição de fluxos assistenciais e instituição de protocolos de rotina recomendados para controle da COVID-19 é imprescindível para a garantia de ambientes de trabalho seguros. Medidas de caráter coletivo e individual devem ser implementadas. Além da oferta de EPI's, devem ser adotadas medidas de reorganização do processo de trabalho, visando minimizar o risco da infecção (Helioterio et al., 2020).

Quanto aos EPIs adequados, a ANVISA recomenda uso de óculos de proteção ou protetor facial, máscara, avental impermeável e luvas de procedimento rotineiramente e, em caso de procedimentos geradores de aerossóis, deve-se usar gorro e máscara N95 (Gallasch, Cunha, Pereira \& Silva-Junior, 2020). Apesar da ANVISA e a National Health Commission of the People's Republic of China indicar o uso de gorro nas diretrizes para proteção contra o novo coronavírus, algumas instituições não fazem a mesma recomendação, como o Centers for Disease Control and Prevention (CDC), que não menciona a utilização de gorros e toucas (equipamento de proteção para cabeça), como EPIs. Sendo assim, percebe-se que ainda não há um consenso estabelecido para a necessidade de equipamentos para a proteção da cabeça contra o COVID-19 (Tristão \& Tavares, 2020).

Vale salientar, que o macacão consiste em outro equipamento de proteção individual para o corpo, entretanto, sua recomendação para a proteção contra o novo coronavírus ainda não é consenso na literatura, visto que algumas instituições o indicam, como o The Korea Center for Disease Control and Prevention (KCDC) e a National Health Commission of the People's Republic of China e outras não, a exemplo da OMS e a Organização Pan-Americana da Saúde, porque apesar de oferecer uma ótima proteção, sua desparamentação é mais difícil de realizar, o que pode predispor a contaminações (Tristão \& Tavares, 2020).

Em relação à importância da paramentação e desparamentação com técnica correta para evitar a contaminação, um estudo demonstrou um checklist para ajudar a lembrar da sequência para tal, o qual se mostrou bastante benéfico, visto que a falta de treinamento para o uso de EPI's é um problema bastante presente. Tal instrumento é dividido em três partes: o que fazer antes da paramentação, durante a paramentação e durante a desparamentação, além disso, contém informações sobre cada EPI, como utilizar, cuidados para reutilizar, sequência de paramentação e desparamentação e outras observações importantes (Braga et al., 2020).

Assim, percebe-se que é preciso atentar não somente a disponibilizar EPIs adequados, mas também como lidar com as dificuldades do seu uso. Um estudo apontou justamente estratégias para lidar com essas dificuldades, como: criação de hábito para uso dos EPIs, divulgar informações sobre a utilização e retirada dos equipamentos de forma correta, criar estratégias para reduzir o incômodo do seu uso e providenciar materiais suficientes (Soares et al., 2020).

Além disso, levando em consideração que o temor em contaminar familiares é uma das maiores preocupações dos profissionais de saúde da linha de frente contra a COVID-19 e isso pode conferir risco para a saúde mental deles, é importante instituir medidas para amenizar tal situação. Algumas medidas sugeridas nos estudos foram: disponibilização de alojamentos para profissionais que moram com os familiares e desejam manter a distancia para evitar a contaminação deles e adoção de transporte seguro para levá-los ao local de trabalho (Helioterio et al., 2020).

Ademais, é de suma importância atentar para monitoramento dos profissionais para identificar os que estão contaminados através de: automonitoramento dos sinais e sintomas, além de afastamento e testes em caso de exposição de alto risco, a exemplo de contato com o paciente sem uso de EPI's (Gallasch, Cunha, Pereira \& Silva-Junior, 2020). Para profissionais de alto risco são fornecidas recomendações mais intensas, como: testagem para COVID-19 quando positivado ter quarentena de 14 dias em um ambiente designado, além disso, os estabelecimentos de saúde devem fornecer apoio psicossocial, bem como atualizar o treinamento de prevenção e controle de doenças (World Health Organization [WHO], 
2019).

\section{Considerações Finais}

Diante do exposto, foi possível verificar que os profissionais de saúde que trabalham na linha de frente contra a COVID-19 sofrem de mais ansiedade, distúrbios do sono e depressão - sendo esse último o distúrbio de maior prevalência quando comparados aos profissionais atuantes na segunda linha e à população geral. Todavia, foi percebido que os enfermeiros foram mais propensos a apresentar distúrbios psicológicos que os médicos. Como fatores de proteção à saúde mental desses profissionais podem ser citados o suporte social dos familiares e dos serviços onde eles atuam. Em contrapartida, dentre os aspectos que afetaram negativamente a saúde mental dos trabalhadores, o principal foi a escassez dos EPIs.

Com efeito, a proteção dos médicos e enfermeiros é de extrema importância pois não só acarreta impacto positivo em sua saúde emocional, mas também física. Assim, dentre os principais EPIs utilizados para a proteção contra a infecção pelo coronavírus rotineiramente encontram-se óculos de proteção ou protetor facial, máscara, avental impermeável e luvas de procedimento e durante a realização de procedimentos geradores de aerossóis, deve-se usar ainda gorro ou toucas e máscara N95. Vale salientar que além dos EPIs, medidas como higienização das mãos, utilização da telemedicina e implementação de barreiras físicas também devem ser adotadas.

Como limitação desta pesquisa, destaca-se a falta de informações sobre o novo coronavírus, visto que é uma doença nova com diversas particularidades ainda desconhecidas, por conseguinte, ainda não há diretrizes padronizadas que tratem sobre a proteção dos profissionais de saúde. Além disso, como o vírus ainda se encontra em circulação e em caráter pandêmico, ele continua afligindo médicos e enfermeiros, assim, a realização de estudos prospectivos que investiguem as alterações comportamentais e emocionais dos trabalhadores deve ser considerada.

\section{Referências}

Adir, Y., Segol, O., Kompaniets, D., Ziso, H., Yaffe, Y., Bergman, I., Hassidov, E., \& Eden, A. (2020) COVID-19: Minimising risk to healthcare workers during aerosol-producing respiratory therapy using an innovative constant flow canopy. European Respiratory Journal, 55(5), 1-3. 10.1183/13993003.010172020 .

Boccalatte, L. A., Larrañaga, J. J., Raffo, G. M. P., Teijido, C. A, Fornari G. G., Staneloni, M. I., \& Figari, M. F. (2020) Brief guideline for the prevention of COVID-19 infection in head and neck and otolaryngology surgeons. American journal of otolaryngology, 1-5. 10.1016/j.amjoto.2020.102484.

Braga, L. M., Siman, A. G., Souza, C. C., Dutra, H. S., Gomes, A. P., \& Siqueira-Batista, R. (2020) Construção e validação do checklist para paramentação e desparamentação dos equipamentos de proteção individual. Revista de Enfermagem do Centro Oeste Mineiro, 10, 1-11. 10.19175/recom.v10i0.4079.

Conegliani, T. V., Uehara, S., \& Magri, M. A. (2020) Prevenção de contágio por covid-19 na exposição ocupacional em saúde: Scoping review TT. CuidArte Enfermagem, 14(2), 156-63.

Couper, K., Taylor-Phillips, S., Grove, A., Freeman, K., Osokogu, O., Court, R., \& Perkins, G. D. (2020) COVID-19 in cardiac arrest and infection risk to rescuers: A systematic review. European Resuscitation Council, 151, 59-66. 10.1016/j.resuscitation.2020.04.022.

DeCastro, T. G., \& Gomes, W. B. (2011). Aplicações do método fenomenológico à pesquisa em psicologia: tradições e tendências. Estudos de psicologia (Campinas), 28, 153-161.

Evanoff, B., Strickland, J., Dale, A., Hayibor, L., Page, E., Duncan, J., Kannampallil, T., \& Gray, D. L. (2020, 08 25) Work-Related and personal factors associated with mental well-being during the COVID-19 response: Survey of health care and other workers. Journal of Medical Internet Research, 22(8). $10.2196 / 21366$

Gallasch, C. H., Cunha, M. L., Pereira, L. A. S., \& Silva-Junior, J. S. (2020, 04 02) Prevention related to the occupational exposure of health professionals workers in the COVID-19 scenario. Revista Enfermagem UERJ, 28, 1-6. 10.12957/reuerj.2020.49596.

Gupta, P. C., Kumar, M. P., \& Ram, J. (2020) COVID-19 pandemic from an ophthalmology point of view. Indian Journal of Medical Research, 151(5), 411418. 10.4103/ijmr.IJMR_1369_20.

Helioterio, M. C., Lopes, F. Q. R. S., Sousa, C. C., Souza, F. O., Pinho, P. S., Sousa, F. N. F., \& Araújo, T. M., (2020) Covid-19: Por que a proteção de trabalhadores e trabalhadoras da saúde é prioritária no combate à pandemia? Trabalho, Educação e Saúde, 18(3), 1-18. /10.1590/1981-7746-sol00289. 
Research, Society and Development, v. 10, n. 15, e38101522307, 2021

(CC BY 4.0) | ISSN 2525-3409 | DOI: http://dx.doi.org/10.33448/rsd-v10i15.22307

Li, S., Wang, Y., Xue, J., Zhao, N., \& Zhu, T. (2020, 03 19) The impact of COVID-19 epidemic declaration on psychological consequences: A study on active weibo users. International Journal of Environmental Research and Public Health, 2032, 1-9.

Malickova, K., Kratka, Z., Luxova, S., Bortlik, M., \& Lukas, M. (2020) Anti-SARS-CoV-2 antibody testing in IBD healthcare professionals: Are we currently able to provide COVID-free IBD clinics? Scandinavian Journal of Gastroenterology, 55(8), 1-3. 10.1080/00365521.2020.1791244.

Miranda, F. M. D., Santana, L. D. L., Pizzolato, A. C., \& Sarquis, L. M. M. (2020) Condições de trabalho e o impacto na saúde dos profissionais de enfermagem frente a covid-19. Revista Cogitare Enfermagem, 25, 1-8. 10.5380/ce.v25i0.72702.

Muller, A.E., Hafstad, E. V., Himmels, J. P. W., Smedslund, G., Flottorp, S., Stensland, S. O., \& Vist, G. E. (2020, 09 01) The mental health impact of the covid-19 pandemic on healthcare workers, and interventions to help them: A rapid systematic review. Psychiatry research, 293, 1-11. 10.1016/j.psychres.2020.113441.

Poersch, A. L., Cardoso, D. L., Ramos, M. Z., Lima, F. M., \& Carvalho, F. G. (2020) Time de resposta rápida em saúde mental (TRRSM): Protocolo de atendimento psicossocial para trabalhadores da saúde no contexto de pandemia. Clinical \& Biomedical Research 40(2), 133-136. 10.22491/2357-9730.103630.

Ribeiro, A. P., Oliveira, G. A., Silva, L. S., \& Souza, E. R. (2020, 06 30) Saúde e Segurança de profissionais de saúde no atendimento a pacientes no contexto da pandemia de COVID-19: Revisão de literatura. Revista brasileira de saúde ocupacional, 45(25), 1-9. 10.1590/2317-6369000013920.

Robertson, L. J., Maposa, I., Somaroo, H., \& Johnson, O. (2020) Mental health of healthcare workers during the COVID-19 outbreak: A rapid scoping review to inform provincial guidelines in South Africa. The South African Medical Journal, 110(10), 1010-1019.

Sanghera, J., Pattani, N., Hashmi, Y., Varley, K. F., Cheruvu, M. S., Bradley, A., \& Burke, J. R. (2020, 10 06) The impact of SARS-CoV-2 on the mental health of healthcare workers in a hospital setting-A systematic review. Journal of Occupational Health, 62, 1-16. 10.1002/1348-9585.12175.

Santana, N., Costa, G. A., Costa, S. S. P., Pereira, L. V., Silva, J. V., \& Sales, I. P. P. M. (2020, 10 30) Segurança dos profissionais de saúde no enfrentamento do novo coronavírus no Brasil. Escola Anna Nery. 24, 1-7. 0.1590/2177-9465-EAN-2020-0241.

Santos, K. O. B., Fernandes, R. C. P., Almeida, M. M. C., Miranda, S. S., Mise, Y. F., \& Lima, M. A. G. (2020, 09 08) Trabalho, saúde e vulnerabilidade na pandemia de COVID-19. Cadernos de Saúde Pública, 36(12), 1-14. 10.1590/0102-311X00178320.

Silva, M. C. N., Neto, F. R. G. X., Lourenção, L. G., Cunha, C. L. F., Santos, J. L. G., Freire, N. P., \& Cunha, I. C. K. O. (2020) Enfermagem e a pandemia da Covid-19: Uma conjugação entre liderança e vulnerabilidade profissional. Enfermagem em Foco, 11(2), 7-8. 10.21675/2357-707X.2020.v11.n2.ESP.4436.

Simms, A., Fear, N. T., \& Greenberg, N. (2020, 05 25) The impact of having inadequate safety equipment on mental health. Occupational Medicine, 70(4), 278-281. 10.1093/occmed/kqaa101.

Soares, S., Souza, N., Carvalho, E., Varella, T., Andrade, K., Pereira, S., \& Costa, C., (2020) De cuidador a paciente: na pandemia da Covid-19, quem defende e cuida da enfermagem brasileira? Escola Anna Nery, 24, 1-7. 10.1590/2177-9465-EAN-2020-0161.

Souza, M. T., Silva, M. D., \& Carvalho, R. (2021, 06 08) Revisão integrativa: o que é e como fazer. Einstein, 8(1), $102-6$.

Sud, S. R. (2020) COVID-19 and keeping clean: A narrative review to ascertain the efficacy of personal protective equipment to safeguard healthcare workers against SARS-CoV-2. Hospital Pediatrics, 570-576. 10.1542/hpeds.2020-0135.

Tristão, F. S., \& Tavares, D. H. (2020) Equipamentos de proteção individual para atendimento de casos suspeitos ou confirmados do novo Coronavírus Journal of Nursing and Health, 10(4), 1-17.

Wańkowicz, P., Szylińska, A., \& Rotter, I. (2020, 08 12) Assessment of mental health factors among health professionals depending on their contact with COVID-19 patients. International Journal of Environmental Research and Public Health, 17(16), 1-9. 10.3390/ijerph17165849.

World Health Organization. (2020). Health workers exposure risk assessment and management in the context of COVID-19 virus. https://apps.who.int/iris/bitstream/handle/10665/331340/WHO-2019-nCov-HCW_risk_assessment-2020.1-eng.pdf.

Xavier, A. R., Silva, J. S., Almeida, J. P. C. L., Conceição, J. F. F., Lacerda, G. S., \& Kanaan, S. (2020, 06 09) COVID-19: Manifestações clínicas e laboratoriais na infecção pelo novo coronavírus. Jornal Brasileiro de Patologia e Medicina Laboratorial, 56, 1-9. 10.5935/1676-2444.20200049. 